\title{
Bioconjugated Silica Nanoparticles: Development and Applications
}

\author{
Lin Wang ${ }^{1}(\bowtie)$, Wenjun Zhao ${ }^{2,3}$, and Weihong $\operatorname{Tan}^{3}(\bowtie)$ \\ ${ }^{1}$ Lilly Research Laboratories, Eli Lilly and Company, Indianapolis, IN 46285, USA \\ ${ }^{2}$ Chinese Academy of Inspection and Quarantine, Huixinli 241, Chaoyang District, Beijing 100029, China \\ ${ }^{3}$ Center for Research at the Bio/Nano Interface, Department of Chemistry and Department of Physiology and Functional Genomics, \\ Shands Cancer Center, UF Genetics Institute and McKnight Brain Institute, University of Florida, Gainesville, FL 32611-7200, USA \\ Received: 17 April 2008/ Revised: 17 June 2008/Accepted: 17 June 2008 \\ (C) Tsinghua Press and Springer-Verlag 2008
}

\begin{abstract}
Advanced bioanalysis, including accurate quantitation, has driven the need to understand biology and medicine at the molecular level. Bioconjugated silica nanoparticles have the potential to address this emerging challenge. Particularly intriguing diagnostic and therapeutic applications in cancer and infectious disease as well as uses in gene and drug delivery, have also been found for silica nanoparticles. In this review, we describe the synthesis, bioconjugation, and applications of silica nanoparticles in different bioanalysis formats, such as selective tagging, barcoding, and separation of a wide range of biomedically important targets. Overall, we envisage that further development of these nanoparticles will provide a variety of advanced tools for molecular biology, genomics, proteomics and medicine.
\end{abstract}

\section{KEYWORDS}

Nanoscience, nanotechnology, silica nanoparticles, bioanalysis, biomedicine

\section{Introduction}

Recently, we have seen the discovery of an increasing number of molecular mechanisms underlying disease processes. Consequently, clinical diagnosis has come to depend heavily on detection and monitoring of individual chemical interactions of smaller and less abundant targets, such as individual cells, mRNA, DNA, proteins, and peptides. Because these targets exist on a nanoscale, probes of equivalent dimensions have been developed, leading to the integration of nanotechnology and biology. This progress, in turn, has led to major advances in medical diagnostics, targeted therapeutics, molecular biology, and cell biology [1-3]. Thus, the adaptation of nanomaterials for biology and medicine has changed the bioanalytical measurement landscape and yielded several promising schemes for widespread use [47]. In addition, the demand for gene profiling and high-throughput drug and disease screening without complex instrumentation or processing steps has driven the development of novel substrates for fast, simple, ultrasensitive, and multiplexed bioanalysis.

Among the different nanomaterials developed for bioanalysis, nanoparticles (NPs) usually form the core of bio-nanomaterials. They can be used as a

Address correspondence to Lin Wang, wang_lin_lw@lilly.com; Weihong Tan, tan@chem.ufl.edu 
convenient surface for molecular assembly and may be composed of inorganic or polymeric materials [8]. In the past decade, a variety of nanomaterials such as quantum dots (QDs) [9, 10], metal NPs [11, 12], lanthanide NPs [13, 14], and silica NPs [15] have each shown their unique properties and been adapted to different applications in the bioanalysis field. Among these, QDs are the most intriguing class of fluorescent probes for cellular imaging and in vivo cell tracking because of their small size (1-10 $\mathrm{nm}$ in diameter), high brightness (20× brighter than most organic fluorophores), good photostability, and multiplexing capability. However, several properties limit the widespread use of QDs. The core of QDs is composed of toxic heavy metals (e.g., cadmium); therefore, cytotoxicity is a definite concern for in vivo applications. QDs are not soluble in water, and must be surface modified with a polymer to allow for use in biological applications. In addition, single colloidal quantum dots emit intermittently (the socalled "blinking" behavior) which is a limiting factor for single particle tracking within cells.

Recently, different fluorescent silica NPs with similar composition and functionalization (known as FloDots and Cornell dots) have emerged as a particularly fascinating fluorescent probe and attracted widespread interest in biology and medicine [15-19]. The variety of chemical and physical modifications possible with silica increases its versatility, and its biocompatibility makes it a relatively benign material. Finally, the silica NP probe is highly hydrophilic and easy to centrifuge for separation, surface modification, and labeling procedures. The silica NPs exhibit enhanced and controllable mechanical and chemical stability and their porosity can also be easily tailored in terms of pore size and organization. Thus, they are superior to polystyrene latex NP probes, which have significant drawbacks. For instance, the hydrophobic property of polystyrene results in easy agglomeration of NPs in aqueous media, and its density $\left(1.05 \mathrm{~g} / \mathrm{cm}^{3}\right)$ leads to difficulties in separation from solution after the surface modification and labeling processes [20].

Highly luminescent silica NPs have been developed for the selective tagging of a wide range of biomedically important targets, such as cancer cells, bacteria, and individual biomolecules [16, 19]. Compared to organic fluorophores, which are the most commonly used bio-labeling reagents, luminescent silica NPs demonstrate the advantages of multiplexing capabilities and ease of functionalization, as well as greater sensitivity and photostability. The advanced optical features of fluorescent silica NP probes are critical for ultrasensitive bio-imaging and detection as well as for real-time tracking and monitoring of complex biological events at the cellular level, which cannot be accomplished using regular fluorescent dyes.

In addition, analysis of complex biological samples typically requires sample pre-treatment. Multi-step procedures, such as solid-phase extraction (SPE), are typically used for sample preparation but they do not remove analytes selectively [21]. In contrast, silica NPs with superparamagnetic properties have been developed as ideal media for the manipulation of trace amounts of bioanalytes from complex biological matrices in applications ranging from detection, separation to targeted delivery for hyperthermia treatment $[15,22]$.

Silica NPs are also promising candidates for improved drug delivery systems. Drug molecules have been loaded into silica NPs, and surface modification of the NPs with biorecognition entities can allow specific cells or receptors in the body to be located [23]. Upon target recognition, NPs can release their drug payload at a rate that can be precisely controlled by tailoring the internal structure of the particles for a desired diffusion/release profile.

It is also possible to make multifunctional silica NPs with the aim of developing nanoscale composites with innovative optical, chemical, and magnetic properties, all combined in one single nanostructure [24, 25]. For instance, magneticfluorescent silica NPs based on iron-oxide NPs combined with fluorophores can be simultaneously manipulated with an external magnetic field and characterized in situ with fluorescence microscopy or confocal scanning microscopy. Such multifunctional magnetic/fluorescent NPs are potentially useful for imaging cells, tissues, and other organs in multiple modes, as well as for the delivery of therapeutic agents to specific targets. 
This article provides an overview of recent progress in silica NP research, with special emphasis on applications in the fields of bio-labeling, imaging, separation, disease diagnostics and therapeutics, and other related technologies. Challenges and perspectives of silica NP probes are also discussed. Although there are other nanomaterials under development, we are unable to cover the entire landscape and therefore only provide the reader with the work being conducted in the silica NP field.

\section{Silica NP synthesis and bionconjugation}

Spherical silica NPs are generally made by one of two synthetic routes: reverse microemulsion or the Stöber method. The reverse micelle or water-inoil (w/o) microemulsion system is composed of a homogeneous mixture of water, oil, and surfactant molecules [26-28]. The single-phase microemulsion system is both isotropic and thermodynamically stable. Water nanodroplets form in the bulk oil phase, which then act as a confined medium (of nanoreactors) for discrete particle formation. Polar and water-soluble dye molecules can be readily encapsulated into silica NPs by this method [29-33] because of the electrostatic attraction of dye molecules to the negatively charged silica matrix. To synthesize organic dye-doped NPs, various trapping methods have been applied, such as introducing a hydrophobic silica precursor [34] or using watersoluble dextran-conjugated dyes under acidic conditions [35]. An advantage of the microemulsion system lies in its typical output of monodisperse and highly uniform NPs [36]. However, fluorophores are physically attached to the silica matrix and may leach out of the particles over time. Furthermore, the use of surfactants necessitates extensive washing to remove the surfactant molecules before any biological application in order to avoid disruption or lysis of biomembranes by the surfactant molecules.

As an alternative, the Stöber method [37] can be applied for synthesizing fairly monodisperse silica particles with diameters ranging from $30 \mathrm{~nm}$ to $2 \mu \mathrm{m}$. In the Stöber process, silica particles are formed by the hydrolysis and condensation of siloxane precursors (e.g., tetraethylorthosilicate (TEOS)) in the presence of ethanol and ammonia. This method has been modified to incorporate a variety of organic dye molecules [38-41] within the silica NPs by covalent attachment. The procedure involves two steps. First, the dye is chemically bound to an amine-containing silane agent (such as 3-aminopropyltriethoxysilane, APTS), and, second, APTS and TEOS are allowed to hydrolyze and co-condense to form dye-doped NPs.

A wide variety of organic dye molecules (Fig. 1) can be incorporated into silica NPs using the Stöber method. The same doping procedure has also been used to incorporate other materials into the silica matrix, such as magnetic NPs of $\mathrm{Fe}_{3} \mathrm{O}_{4}$ and $\mathrm{Fe}_{2} \mathrm{O}_{3}$ or luminescent quantum dots $[29,42,43]$. Magnetic NPs or quantum dots are used as seeds for hydrolysis and condensation of TEOS, resulting in silica-coated NPs. In addition, mesoporous silica NPs [44] have also been prepared by a surfactant (e.g., cetyltrimethylammonium bromide (CTAB), templated co-condensation method using TEOS and organosiloxane precursors [45], or they can be formed by reacting diglyceroxysilane in ethanol and
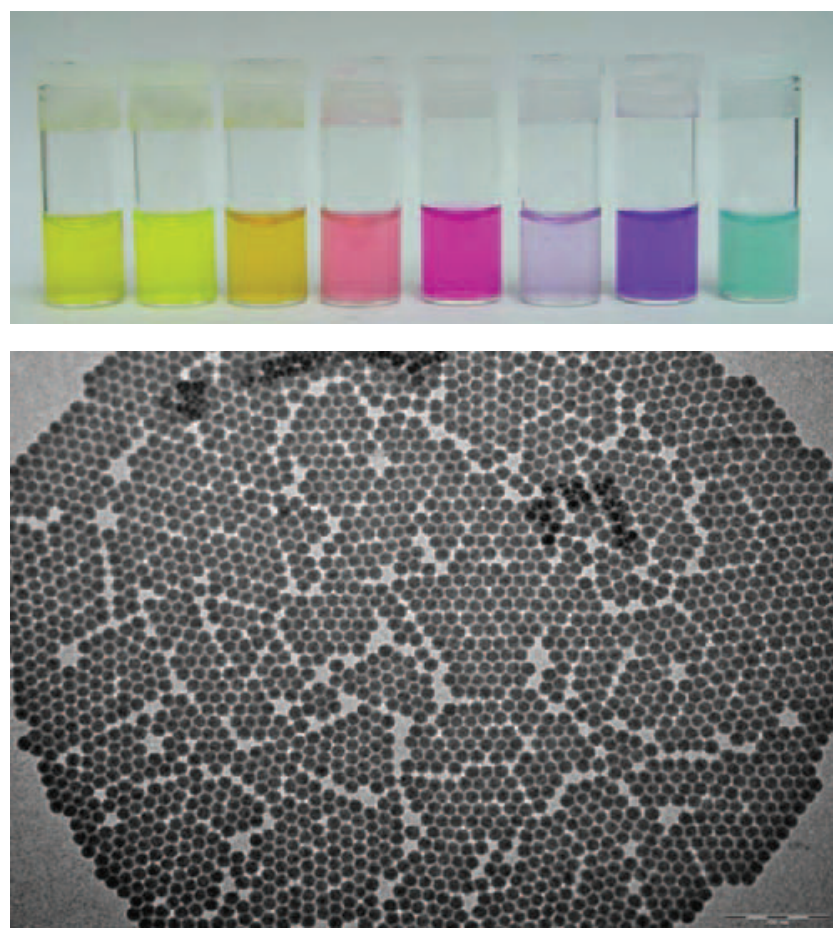

Figure 1 Fluorescent silica NP architecture may be expanded to incorporate a wide range of dye molecules including (left to right): fluorescein isothiocyanate, Oregon green, rhodamine, rhodamine $6 \mathrm{G}$, tetramethylrhodamine, Texas red, 6-carboxyl-X-rhodamine, and cyanine Cy5. A representative TEM image shows the NP size is $40 \mathrm{~nm}$ in diameter 
methanol-free conditions in pure water or in buffer solutions [46].

Once NPs have been formed, an additional layer of linker molecules with various reactive functional groups (e.g., amine, thiol, carboxyl or methacrylate) is often attached. This is typically achieved by applying an additional silica coating (post-coating) that contains the desired functional group(s). In addition to providing the reaction sites for bioconjugation, the functional groups also change the colloidal stability of NPs in solution. For example, inert negatively charged organosilane compounds containing phosphonate groups can be introduced into NPs during post-coating [47]. This step increases the repulsive forces between the particles in solution and thus improves long-term NP stability. In addition, polyethylene glycol (PEG, a neutral polymer) can also be introduced to the NP surface in order to reduce nonspecific binding by inhibiting the adsorption of undesired charged biomolecules [48].

After NPs have been modified with different functional groups, they can act as a scaffold for the grafting of biological moieties, such as biocompatibles (e.g., dextran, antibodies, DNA or peptides), by means of standard covalent bioconjugation schemes [49] (Fig. 2), such as carbodiimide chemistry, disulfide-coupling chemistry and succinimidyl ester hydrolysis chemistry.

In addition to covalent binding to the NP surface,

(a)

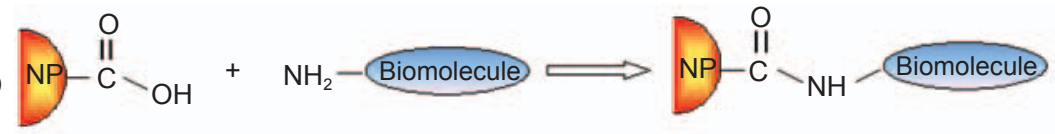

(b)

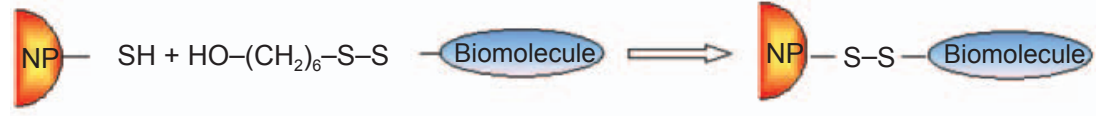

(c)

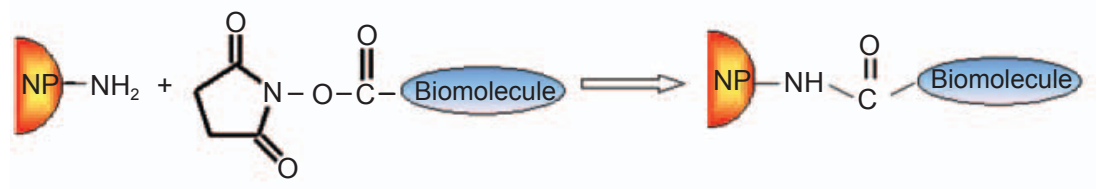

(d)

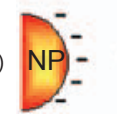

Figure 2 Representative bioconjugation schemes for the attachment of biomolecules onto the surface of silica NPS bio-recognition entities can also be physically adsorbed onto the NP surface using electrostatic interactions between NPs and charged adapter molecules [50,51] or between NPs and proteins modified to incorporate charged domains. However, covalent binding of biomolecules to NPs is preferred, not only to avoid desorption from the NP surface, but also to control the number and orientation of the immobilized biorecognition entities.

\section{NP characterization}

Characterization of NPs typically includes measuring the particle size, surface charge, surface functionalitiy, and optical and magnetic properties. Transmission electron microscopy (TEM) and scanning electron microscopy (SEM) are commonly used to evaluate the NP size in the vacuum state [40,41]; light scattering techniques are applied to measure NP size in aqueous media while providing NP size distribution and relative dispersion [52].

The zeta potential of a particle indicates the overall charge that the particle acquires in a particular medium. The resulting repulsive force can be used to predict the colloidal stability of the NP product. If all the particles in suspension possess a large positive or negative zeta potential, they will repel each other, thus avoiding NP agglomeration. Conversely, low zeta potential values result in the tendency of NPs to flocculate, or coagulate loosely. Therefore, surface modification is usually performed in order to increase the repulsive forces between the particles in solution and hence improve the long-term stability of NPs [53]. Furthermore, the effect of varying the $\mathrm{pH}$, concentration of an additive, or the ionic strength of the medium on the zeta potential and rheological properties can give information which helps in formulating the NP product to give maximum stability.

The application of dyedoped silica NPs for bioimaging and labeling depends on their 
optical properties, and there are several advantages of fluorescent NPs over commonly used organic dyes. First, better sensitivity can be achieved by incorporating a large number of dye molecules in a single NP system. For example, tetramethylrhodamine (TMR) dye-doped silica NPs (60 $\mathrm{nm}$ in diameter) can each emit an extremely strong fluorescence signal (up to $10^{4}$ times that of a TMR fluorophore) [54], achieving high signal amplification for ultrasensitive target detection and for monitoring rare events that are otherwise undetectable with conventional fluorophore labeling strategies. Secondly, because of the protective encapsulation of fluorophores by the host silica material, increased photostability is generally attained in NPs. This feature makes NPs particularly useful as fluorescent markers in complex biological environments, where it is often desirable to observe markers for extended periods of time against the background of intrinsic cellular emissions [31]. Moreover, the potential of preparing NPs with any existing single or multiple fluorophore(s) gives NPs a unique versatility for various applications, including multiplexed bioanlaysis [55]. Finally, whereas organic fluorophores require customized chemistry for conjugation of biomolecules to each fluorophore, a universal approach can be used for conjugating biomolecules to all silica NPs.

\section{Applications of silica NPs}

The advanced features of silica NP probes give them a substantial advantage for use in the detection of single molecules, bioimaging and extraction of cells and cellular components, as well as disease targeting. The scope of their applications ranges from biology to medicine.

\subsection{Cancer cell imaging}

For effective cellular labeling techniques, biomarkers need to have excellent specificity toward biomolecules of interest and also have optically stable signal transducers. Immunofluorescent labeling of both cancer cell surface marker and tissue sections by dyedoped silica NPs has demonstrated high specificity and high intensity, thus making direct observation for instant diagnosis of pathological samples during surgical procedures a possibility [56]. Different strategies have been explored for using NP probes to target cancer cells.

First, affinity and specificity associated with the antibody-antigen recognition have been widely explored in developing immunoassays. Primary or secondary antibodies are covalently immobilized onto the NP surface in order to selectively and efficiently bind various cancer cells [31, 57, 58]. In one demonstration, a mouse anti-human CD10 antibody was used as the recognition element on NPs (diameter: $63 \mathrm{~nm} \pm 4 \mathrm{~nm}$ ). The mononuclear lymphoid cells were incubated with the CD10 NPs and then washed with phosphate buffered saline (PBS) to remove unbound NPs. Fluorescence microscopy was then used to image the leukemia cells. The brightly fluorescent cells bound with NPs were easily detected under the fluorescence microscope. Control experiments without antibody and specificity studies with nontarget cells showed the effectiveness of this method in the selective detection of leukemia cells [31].

Aside from antibody-mediated recognition, other affinity reagents, such as receptor ligands and recognition peptides, can also be attached onto NPs in order to label cell-membrane proteins. For instance, folic acid, a small vitamin molecule recognized by cancer cells, was attached to dye-doped silica NPs and targeted to SCC-9 cancer cells, which overexpress folate receptors [59]. Laser scanning confocal images demonstrated that folate-fluorescent NPs were internalized via a receptor-mediated endocytosis process by the SCC-9 cells in much larger amounts compared to the normal human dermal fibroblast cells, thus confirming the efficacy and robustness of the detection system for human squamous cancer detection.

Peptide-targeted uptake is another efficient technique for cancer cell imaging. This technique is based on the propensity of the cells to recognize and internalize NPs labeled with specific peptides (e.g., HIV-derived TAT peptide), and even deliver them to specific cellular compartments, such as the nucleus. Human lung adenocarcinoma (A549) cells (in vitro) and rat brain tissue (in vivo) were successfully labeled using TAT-labeled NPs (70 nm in diameter). Using this strategy, diagnostic and therapeutic agents can be 
delivered to the biological target of interest [47].

Recently, aptamers have emerged as a novel class of ligands. Aptamers are short strands of DNA/ RNA for recognition of a variety of targets including proteins and small molecules as well as complex samples. Aptamers have significant advantages over antibodies and peptides, including high affinity, excellent specificity, and lack of immunogenicity [60]. Aptamers can be prepared at lower cost than antibodies via automated synthesis, which allows directed modifications for further chemistry and engineering (e.g., immobilization functionalities) onto silica NPs. Specific targeting and visualization of acute leukemia cells with aptamer-conjugated NPs have been developed using fluorescence microscopy or flow cytometry [61, 62]. Compared with fluorophore-aptamer conjugates, NP-aptamer conjugates greatly increase the fluorescence signal from the cell. This property shows the potential applications of NPs in the elucidation of cells with low densities of aptamer binding sites, or with relatively weak binding probes where the fluorescence signal from the fluorophore is too weak for observation, where as the strong NP signal is easily detectable. Therefore, NPs can significantly improve our ability to perform cellular imaging and targeting.

\subsection{Ultrasensitive single bacterium detection}

The rapid and accurate detection of trace amounts of organisms, such as pathogenic bacteria, is important for food and water safety, clinical diagnosis, and the prevention of accidental outbreaks. In the case of intentional terrorist acts, early detection of trace amounts of pathogenic microorganisms is critical. However, current bacteria detection methods rely on the ability of microorganisms to grow into visible colonies over time in special growth media, which may take 1-5 days. Under these conditions, detection of trace amounts of bacteria, which typically requires amplification or enrichment of the target bacteria in the sample, would require an even greater amount of time for detection. Thus, time constraints and difficulty of on-site analysis present major obstacles to optimal bacteria detection. To overcome these limitations, a new type of NP-based bacteria detection method has now been developed [35]. Specifically, antibodies against E. coli $\mathrm{O} 157: \mathrm{H7}$ were conjugated to dye-doped silica NPs (diameter: $60 \mathrm{~nm} \pm 4 \mathrm{~nm}$ ). By varying the doping ratio of the three tandem dyes, fluorescence resonance energy transfer (FRET)mediated emission signatures were tuned, and the NPs (diameter: $70 \mathrm{~nm} \pm 5 \mathrm{~nm}$ ) exhibited different colors under single wavelength excitation $(488 \mathrm{~nm})$, forming NP-antibody conjugates or nanoprobe complexes. Under these conditions, the NP-antibody conjugates specifically associate with E. coli O157:H7 cells and generate high intensity fluorescence signals in $30 \mathrm{~min}$, but not with E. coli $\mathrm{DH} 5 \alpha$ cells, which lack the surface O157:H7 antigens. The dye-doped NPs are hundreds of times smaller than bacteria; thus, by conjugating the NPs with antibodies that specifically recognize the bacterial surface antigens, as many as $10^{4}$ NPs can bind to the surface of each bacterial cell. Such high numbers of surface-bound NPs should generate sufficient fluorescence signal for detection, thereby achieving ultrasensitive detection of bacterial cells. Furthermore, since this method does not require an amplification or enrichment step, the bacterial detection process can be adapted to realtime detection.

In order to adapt the NP-based assay for routine use in bacteria detection, high-throughput determination of bacteria samples is critical. To determine large numbers of bacteria, the singlebacterium assay noted above can be easily adapted by testing many aliquots of samples simultaneously with a plate reader fluorometer [35]. More than 300 samples can be detected with a single-bacterium detection limit. This method is capable of determining the existence of a single bacterium with $99.99 \%$ accuracy when compared to the widely used colony forming units/ $\mathrm{mL}$ count (CFU) on agar plates. The results were further confirmed using a laboratorymade flow cytometer which precisely detected single bacterial cells with antibody-conjugated NPs bound to them and recoded the fluorescence spike when the cells flowed through the detection channel [63]. The total time for the sample detection and analysis with this flow system was only a few minutes, which minimized the duration of the bacteria assay even further.

To test the utility of the method for bacteria 
detection in real samples, the number of E. coli O157:H7 in several spiked ground beef samples was determined. The results confirmed that a single bacterium recovered from a ground beef sample could be effectively detected, and the effects of potential interferences, such as fat in the ground beef, were negligible [35].

\subsection{DNA and microarray detection}

DNA and oligonucleotide microarrays have revolutionized gene expression profiling by allowing highly parallel and quantitative monitoring of gene transcripts from an organism [64]. This advance has made it possible to tackle qualitatively different questions in biology and medicine. Despite the extensive profiling and genotyping capability of microarrays, one of the difficulties of this technology is accurate detection of low-abundance targets. This problem can be solved by either enhancing the detection sensitivity or increasing the fluorescence signal.

In order to increase the fluorescence signal of the gene target, fluorescent silica NPs have been exploited as probes for DNA/microarray detection. The first lab-based trial was based on a sandwich assay [65]. Capture DNA was immobilized onto a glass surface and hybridized with unlabeled target DNA complementary to both the capture and probe DNA sequences. NPs with attached probe DNA were then added. One probe DNA hybridizes one target DNA and thus immobilizes one dye-doped NP (60 nm in diameter) on the surface, bringing tens of thousands of dye molecules for signaling (Fig. 3). Using this strategy, the DNA detection limit can reach sub-picomolar levels. Single nucleotide polymorphism detection is also feasible. Cyanine Cy3- and Cy5-doped Au/silica core-shell particles have also been prepared and used in two-color microarray detection in a sandwich assay format [66]. The developed NPs have a core-shell structure containing $15 \mathrm{~nm}$ Au colloidal cores with 95 dyealkanethiol (dT) 20 oligomers chemisorbed on each $\mathrm{Au}$ particle surface and silica coatings (10-15 nm) bearing thiol functional groups. Use of these dyedoped NPs resulted in higher sensitivity with a detection limit of $1 \mathrm{pmol} / \mathrm{L}$ for target DNA. An order of magnitude increase in sensitivity enhancement and extension of dynamic range was achieved by using these NPs as labeling reagents in microarray detection compared with fluorophores.

The NP-based DNA detection strategy can be extended to the use of NPs as fluorescent labels for DNA and protein microarray technology in order to meet the critical demand for enhanced sensitivity [48]. Tris(2,2'-bipyridyl)dichlororuthenium(II) hexahydrate (RuBpy)-doped silica NPs (60 nm in diameter, each containing $>10000$ dye molecules) have been used as highly sensitive and photostable labels in Affymetrix GeneChips technology. As an illustration, biotinlabeled cRNA samples from a human lung cancer cell line were hybridized on the arrays and then incubated with streptavidin, followed by washing and staining with PEG-biotin-labeled NPs. Even with the present unfavorable imaging modality and existing optical excitation and detection systems of the GeneChips, the fluorescent silica NPs were demonstrated to be superior to the traditional streptavidin-phycoerythrin (SAPE) staining probe used under similar conditions in terms of giving a simplified staining procedure, higher photobleaching threshold, and enhanced

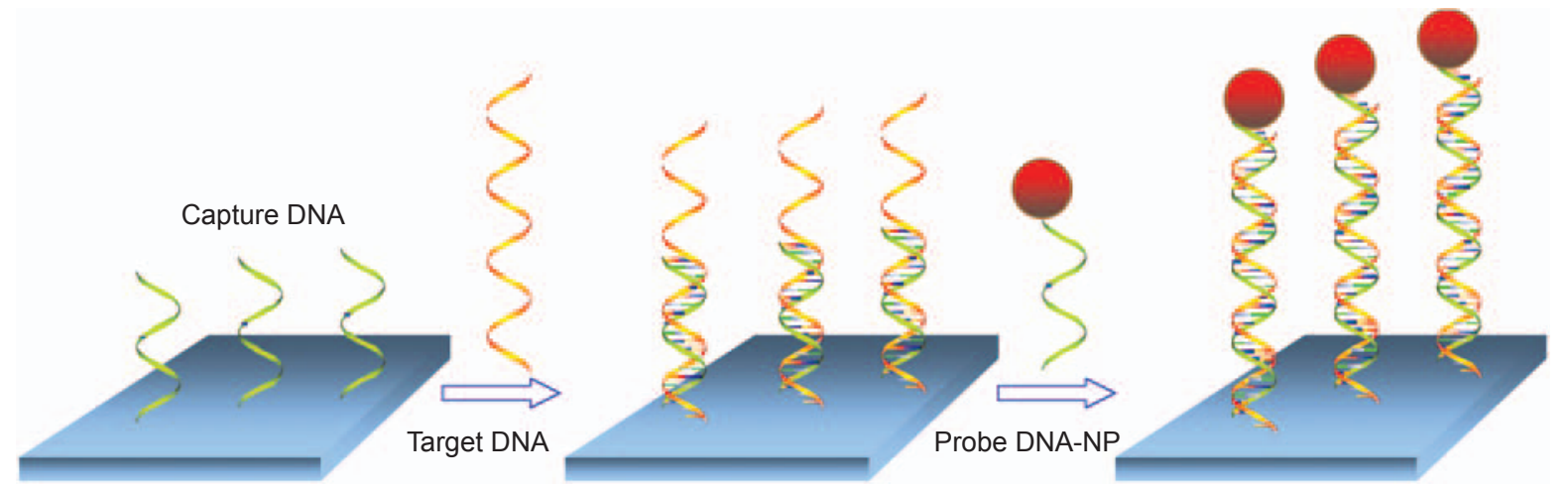

Figure 3 Schematic representation of a sandwich DNA assay based on bioconjugated silica NPs 
luminescence signal. Having achieved a concentration detection limit of $50 \mathrm{fmol} / \mathrm{L}$, the work demonstrated the ability of using luminescent NPs as probes for commercial microarray systems, making them less costly, more reproducible, and potentially quantitative than conventional methods.

\subsection{Barcoding tags}

The drive to understand biology and medicine at the molecular level coupled with the need for accurate quantitation demands high-throughput bioanalysis. As our understanding of diseases like cancer and arteriolosclerosis becomes more detailed, the number of identified targets (proteins or oligonucleotides) that indicate clinical outcome also increases. A typical diagnostic result requires screening for numerous particular target molecules in a single sample. Multiplexed analysis, whereby numerous targets can be detected at the same time, is therefore the method of choice for efficiently deciphering the amount, relationship or structure of multiple analytes simultaneously in a biological sample. Simultaneous tracking of a panel of molecular markers will allow scientists to understand, classify and differentiate complex human diseases. This goal is, however, confounded by the use of conventional fluorophores (each with a spectral width of 50-200 $\mathrm{nm}$ ) since only 4 to 6 different fluorescent dyes can be accommodated without overlapping in the visible region of 400 to $800 \mathrm{~nm}$, thereby greatly limiting the multiplexing capability.

Recently, however, dye-doped silica NPs have been used as novel substrates for multiplexed optical encoding [33,67]. By encapsulating multiple fluorophores simultaneously into the NPs at precisely controlled ratios, multiplex analysis becomes feasible. For instance, two inorganic dyes, tris $\left(2,2^{\prime}-\right.$ bipyridine)rutheniu(II) chloride hexahydrate (RuBpy) and tris(2,2'-bipyridyl)osmium(II) bis(hexafluoro phosphate) (OsBpy), were simultaneously doped into NPs at precisely controlled ratios using the microemulsion process [67]. These two dyes have similar structures and share a broad overlapping excitation spectrum, but have distinct maximum emission wavelengths at 610 and $710 \mathrm{~nm}$, respectively. Therefore, encapsulating the two dyes at precisely controlled ratios in NPs yields unique barcoding signatures, and a single light source is sufficient for reading all the dual-dye-encoded NPs (diameter: $70 \mathrm{~nm} \pm 3 \mathrm{~nm}$ ). Figure 4 displays a schematic representation of the dual-fluorophoreNP encoding system and its multiplexing potential. The antibody-conjugated NPs with varying intensity ratios were mixed with a cocktail of bacteria. When the bacteria-NP complex flows through a channel, such as a flow cytometer, each bacterium-NP complex will exhibit the unique fluorescence signature of the attached NPs (Fig. 4). Rapid, sensitive, and selective bacteria detection can be achieved in this manner.

Furthermore, in order to generate more versatile

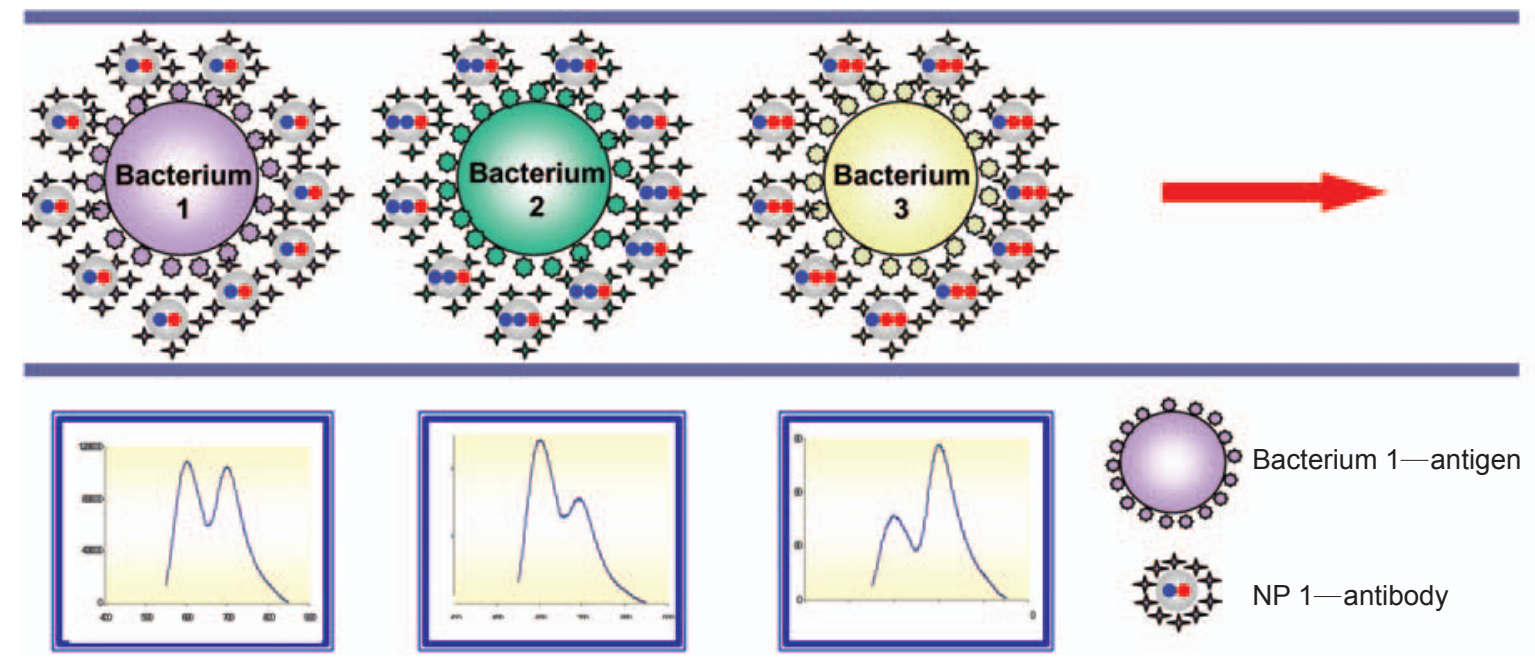

Figure 4 Schematic representation of the dual dye-encoding system and multiplexing potential. As the bacteria pass through the channel, the fluorescence signals correspond to the different encoded NPs specifically attached to the target bacteria 
colors and improve the quantum yield, three energy transfer organic fluorophores were also coencapsulated inside silica NPs. The three tandem dyes were carefully chosen to allow for efficient fluorescence energy transfer. Fluorescein isothiocyanate (FITC), rhodamine 6G (R6G), and 6-carboxyl-X-rhodamine (ROX) were employed because of their effective spectral overlapping. By varying the doping ratio of the three tandem dyes, fluorescence resonance energy transfer (FRET)-mediated emission signatures were tuned, and the NPs (diameter: $70 \mathrm{~nm} \pm 5 \mathrm{~nm}$ ) exhibited different colors under single wavelength excitation (488 $\mathrm{nm}$ ), which favors multi-color imaging applications [33] (Fig. 5(a)). For example, three kinds of multi-color FRET NPs were conjugated to monoclonal antibodies specific for three pathogenic bacteria species (E. coli, Salmonella typhimurium, and Staphylococcus aureus). These antibody-NPs were incubated with small concentrations of the bacteria. After the incubation and washing steps, simultaneous and sensitive detection of the multiple bacterial targets was achieved [68] (Fig. 5(c)). The SEM image in Fig. 5(b) confirms the successful binding between the NPs and the Salmonella typhimurium bacteria.

Additionally, it is noteworthy that the covalent triple-dye labeling approach can be further extended to any energy transfer dye series allowing the generation of a large FRET NP barcoding library. It is very probable that such multiplexing technologies will allow the advantages of NPs to be combined with those of microfluidics and microarrays. In addition, non-invasive molecular imaging technologies could be developed using these NPs by taking advantage of the large Stokes shift of FRET NPs which facilitates detection in samples with significant light scattering or endogenous fluorescent compounds. This should allow viral particles to be followed in vivo, drug molecules to be analyzed in biological systems, and tumor cells to be tracked in real time. In order to be widely used in these areas, however, more strategies need to be established to minimize false positives or false negatives during multiplexing, and more stringent control of the amount of doped dye is required to ensure reproducibility.

\subsection{Separation and purification of biological molecules and cells}

In recent years, magnetic NPs have been proposed for biomedical use $[15,69]$. The fact that a magnetic field gradient can be used to either remotely position or selectively filter biological materials leads to a number of separation and purification applications. For example, magnetic silica NPs were developed for collection of trace amounts of DNA/RNA molecules from a complex mixture [43]. The NPcomplex was fabricated with a magnetic NP, a silica layer, a biotin-avidin linkage, and a molecular beacon [70, 71] DNA probe. The magnetic NPs serve as magnetic carriers and the molecular beacon probes act as recognition elements and indicators for specific gene sequences. The melting profiles of the molecular beacon on the NP surface allow for efficient isolation of the target DNA from single base mismatched DNA. By varying the temperature

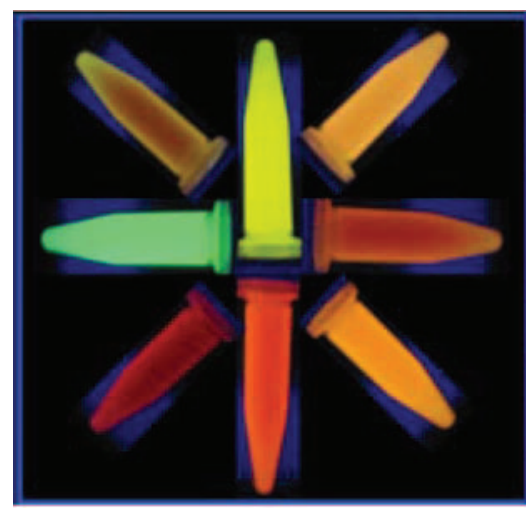

(a)

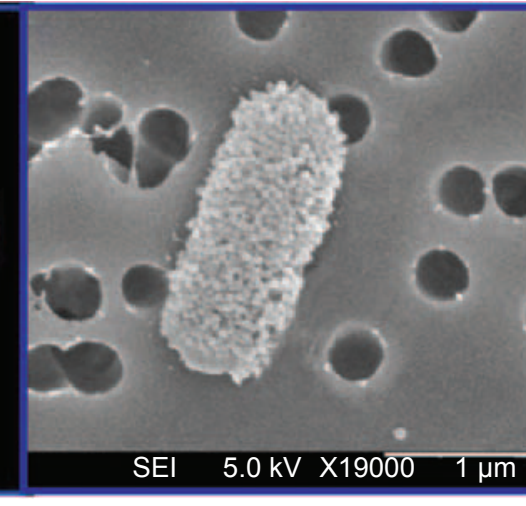

(b)

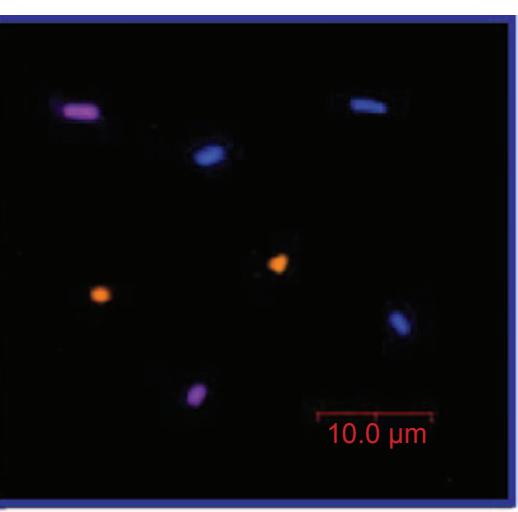

(c)

Figure 5 (a) FRET NP samples with different doping dye concentrations. (b) SEM image of a Salmonella typhimurium (ST) bacterium coated with anti-ST-IgG NPs. (c) Confocal image of three bacteria species (E. coli, Salmonella typhimurium, and Staphylococcus aureus) specifically covered with three types of monoclonal antibody-labeled NPS

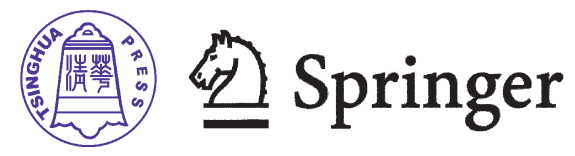


and separating the mixture by magnetization, the NP-complex is able to separate trace amounts of target DNA/RNA in a complex mixture with high specificity and excellent collection efficiency. The separation and collection of trace amounts of single base mismatched DNA could potentially be an effective way to detect mutant cancer genes and prevent cancer before it occurs.

In another application, aptamer-labeled magnetic silica NPs, along with fluorescent silica NPs, were explored for the collection, isolation, and detection of intact leukemia cells from mixed cell and wholeblood samples [61]. Both fluorescent and magnetic NPs were modified with an aptamer selective toward CCRF-CEM leukemia cells. Magnetic NPs were used for selective capture and magnetic collection; fluorescent NPs were used for fluorescence sensing. In as little as $5 \mathrm{~min}$, the leukemia cells were magnetically separated and selectively stained. The two-particle assay was also able to capture leukemia cells from a spiked blood sample. The method has been extended to multiple cell collection, where three types of cancer cells were selectively collected and detected [62]. Figure 6 displays a schematic diagram of the multiple cell extraction procedure employed. Fetal bovine serum was spiked with three cell types for the corresponding cell extraction experiments. The cell types were analyzed using the aptamerconjugated magnetic NPs and fluorescent NPs. The stepwise extraction was performed by adding the specified amounts of magnetic NPs for Ramos cells, followed by CEM aptamer-conjugated magnetic NPs, and finally Toledo-specific magnetic NPs. Each set of magnetic NPs was separately incubated with the cell samples. The magnetic extractions of each step were redispersed in cell media, followed by incubation with the CEM aptamer-conjugated fluorescent NPs, and the magnetic extraction procedure was performed. Similarly, the Toledo and Ramos aptamer-conjugated fluorescent NPs were subsequently introduced to their respective samples. The confocal images (Fig. 6) show the extraction of all three cells treated with all the aptamer-conjugated NPs. The result demonstrates the wide applicability of this methodology for medical diagnostics and cell enrichment and separation.

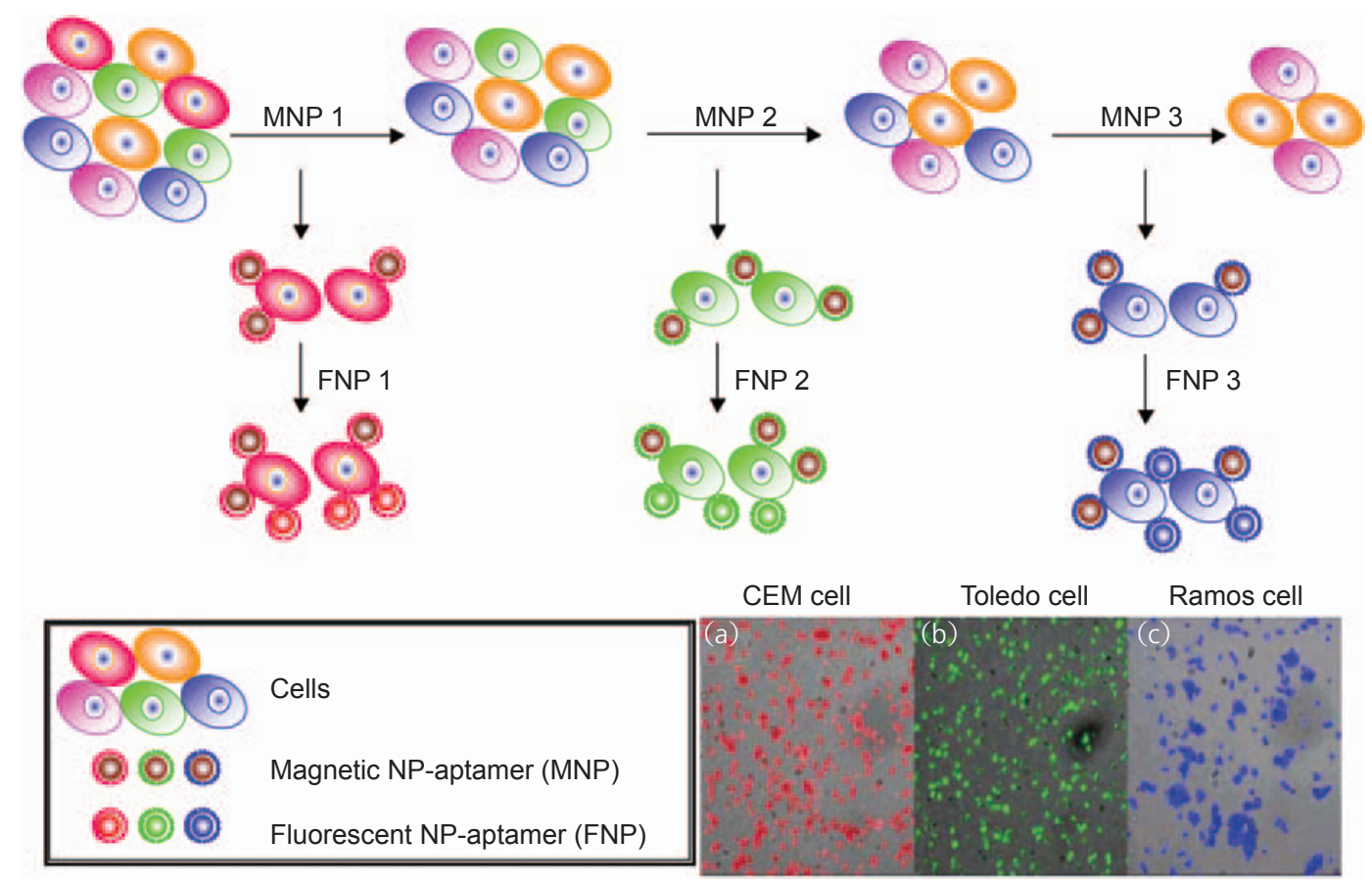

Figure 6 Schematic representation of the multiple extraction procedure from fetal bovine serum with the magnetic NPs being added and extracted stepwise and the corresponding fluorescent NPs (tris $\left(2,2^{\prime}\right.$ bipyridyl)dichlororuthenium (II) hexahydrate (RuBpy), tetramethylrhodamine (TMR), and cyanine Cy5 NPs) being added after magnetic extraction of cell samples. The fluorescence images display the extracted cell samples stained with specific aptamer-conjugated fluorescent NPs 


\subsection{Gene/drug delivery}

It is only one step from nanotechnology to nanomedicine, which may be defined as the monitoring, repair, construction, and control of human biological systems at the molecular level, using engineered nanodevices and nanostructures. A key issue in the practice of this novel science is the proper distribution of drugs and other therapeutic agents within a patient's body. Because of their ability to control drug release and target particular organs/tissue, to act as carriers of oligonucleotides in antisense therapy, and to deliver proteins, peptides, and drugs, silica NPs have attracted considerable attention in the biomedical field.

For example, fluorescent silica NPs can act as nonviral vectors for gene delivery and biophotonics methods may be used to optically monitor intracellular trafficking and gene transfection. The potential of cationic silica NPs (pure silica NPs without surface modifications do not seem to be able to condense and deliver DNA) was investigated for in vivo gene transfer [72]. The NPs were tested for their ability to transfer genes in vivo in the mouse lung, and a two-fold increase in the expression levels was found with silica particles in comparison to enhanced green fluorescent protein (EGFP) alone. Very low or no cell toxicity was observed, suggesting that silica NPs have genuine potential as candidates for gene transfection. Another example involves organically modified mesoporous silica NPs which were used for in vivo gene delivery to the brain. It has been shown that these amino group-functionalized NPs not only bind and protect plasmid DNA from enzymatic digestion but also transfect cultured cells and deliver DNA to the nucleus [73]. Moreover, the gene delivery process does not cause the tissue damage or immunological side effects that have been commonly observed with viral-mediated gene delivery [74, 75].

Silica NPs are also promising candidates for improved drug delivery systems because of their intrinsic hydrophilicity, biocompatibility, and nontoxicity, as well as the excellent protection they provide for their encapsulated drugs. With drug molecules loaded into silica NPs, surface modification of the NPs with biorecognition entities can allow specific cells or receptors in the body to be located. Upon target recognition, NPs can then release their drug payload at a rate precisely controlled by tailoring the internal structure of the particles according to a desired diffusion (release) profile [23]. Furthermore, after the NPs are efficiently taken up by tumor cells, irradiation of the photosensitizing drug entrapped in the NPs results in efficient generation of singlet oxygen, which, in turn, causes significant cancer cell damage [73].

Interestingly, the discovery that mammalian cells take up and internalize mesoporous silica NPs without cytotoxic effects opened the door to the use of these materials as a drug delivery system [76]. The high surface area $\left(>900 \mathrm{~m}^{2} / \mathrm{g}\right)$, tunable pore diameter (2-20 nm) and uniform mesoporous structure (hexagonal channels or cubic pores) of the mesoporous silica NPs offer unique advantages for loading and releasing large quantities of biomedical agents. Mesopores loaded with guest molecules were capped by inorganic NPs, or large organic molecules, via a chemically cleavable disulfide linkage to the mesoporous NP surface. Since drug molecules are effectively physically trapped, they are unable to leach out of the mesoporous NP host (average particle size of $200.0 \mathrm{~nm}$ and an average pore diameter of $2.3 \mathrm{~nm}$ ), thus preventing any premature release. In this case, the release was finally triggered by exposing the capped mesoporous NPs to chemical stimulation that could cleave the disulfide linker, thereby removing the NP caps and releasing the poreentrapped drug molecules. Compared with many current biodegradable polymer-based drug delivery systems that rely on the hydrolysis-induced erosion of the carrier structure, the mesoporous NP structure provides the ability to release the cargo in a controlled manner [77]. However, thiol oxidation may lead to cell death, which is a concern for in vivo applications.

Magnetic silica NPs for gene delivery and drug targeting have also been effectively demonstrated in animal models. Once the magnetic NPs have been taken up by a tumor, an alternating magnetic field is applied that couples to the particles, resulting in efficient local heating [78]. Apoptosis may be induced, and cells are destroyed thus increasing the efficacy of either chemotherapy or radiotherapy. 


\subsection{Multifunctional silica NPs}

In clinical trials today, it is common to use one set of agents for diagnosis and another set of agents for therapeutics and treatment. We should ask whether it is possible to combine these two procedures into a single modality that would improve our ability to achieve diagnosis and treatment, and even monitoring in real time. It is this integration of nanochemistry, targeting systems, and biotechnology that has permitted the fabrication of multifunctional NP probes for bioimaging, diagnostic, and therapeutic purposes. Multifunctional NPs in general have at least a tripartite constitution, featuring a core constituent material, a therapeutic and/or imaging payload, and biological surface modifiers, which enhance the biodistribution and tumor targeting of the NP dispersion (Fig. 7). Recently, multifunctional magnetic and fluorescent NPs have been developed that consist of a thin silica shell encapsulating magnetic NPs as well as fluorescent dyes [24, 79, 80]. These NPs ( $100 \mathrm{~nm} \pm 10 \mathrm{~nm}$ in diameter) are potentially useful in a variety of areas because they can be simultaneously manipulated with an external magnetic field and characterized in situ by optical spectroscopy. For example, multifunctional fluorescent, radio-opaque and paramagnetic silica NPs have been synthesized for multifaceted bioimaging applications [81]. In addition, nearinfrared (NIR) fluorescent and magnetic NPs (32 nm in diameter) have been successfully applied in dual labeling of brain tumors which could help surgeons during the preoperative planning phase and during surgical resection of tumors [82]. Moreover, when the NP surface was functionalized with a biotargeting group, such as leutinizing hormone-releasing hormone, the NPs targeted receptor-specific cancer cells [83]. Subsequent exposure to a DC magnetic field resulted in the selective destruction of the cancer cells. This study showed that multifunctional NPs are potential diagnostic and therapeutic tools for cancer and infectious diseases. Using multifunctional NPs, it is possible to combine the ability to capture, separate, visualize, and treat cells (or proteins) within one reagent.

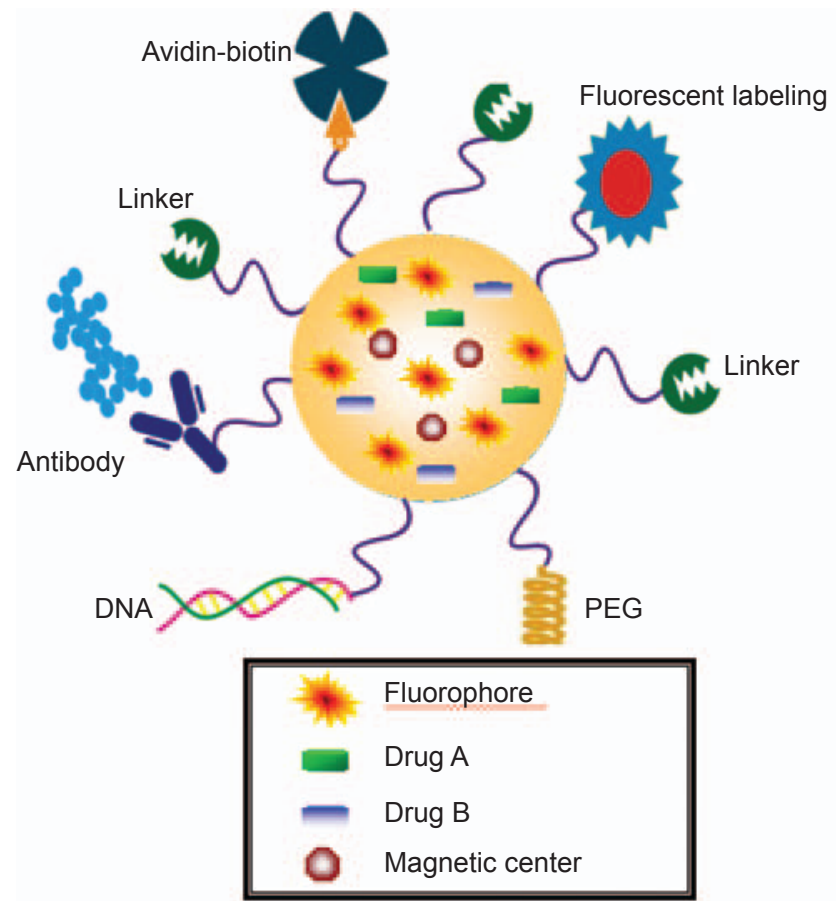

Figure 7 Multifunctional NPs with the ability to carry one or more therapeutic agents: biomolecular targeting through one or more conjugated antibodies or other recognition agents; imaging signal amplification, by way of doping fluorophores or other contrast agents; and biobarrier avoidance, exemplified by polyethylene glycol (PEG) to avoid macrophage uptake

\section{Challenges and trends}

Although researchers have made advances using silica NPs in bioimaging, labeling, separation, disease diagnosis, and therapy, several obstacles and limitations still exist. First, dye leakage from the fluorescent silica NPs over time needs thorough investigation. This phenomenon decreases particle brightness, increases background signal, and exposes the dyes to their environment. Second, nonspecific binding of silica NPs has not yet been resolved. Control strategies need to be established for improving NP dispersion in solution to prevent agglomeration, decrease background noise, and reduce nonspecific adhesion to surfaces. In addition, for ultrasensitive analysis to be achieved, an optimal number of dye molecules need to be doped inside one NP. However, there is always a compromise between NP size and the brightness. Large and bulky sized NPs may block some biological binding sites in close proximity or limit the use of NPs in certain 
applications, such as the FRET-based investigations. It has been suggested that NP-mediated cellular response is size-dependent and that 40 to $50 \mathrm{~nm} \mathrm{NPs}$ form the critical cutoff point for receptor-mediated internalization [84]. Therefore, the balance between size and intensity must be optimized for different applications.

Silica NPs have found broad and successful applications in a variety of in vitro bioassays. They have also found practical implementation in the biomedical field for diagnosis with proper and efficient delivery of pharmaceuticals. However, applications of these tools in medical research and clinical practice require further development. This is illustrated by the cytotoxicity of silica NPs, which must be fully investigated before considering the intravenous injection of silica NPs into diseased tissues and organs in the human body. For drug delivery, there are barriers to controlling the interaction of NPs within the body before this technology can be effectively translated into practical therapeutic regimens. Methods of targeting NPs to specific sites of the body while avoiding capture by organs, such as the liver and spleen, must also be addressed. Currently, however, few data on silica NP toxicity exist. On the one hand, colloidal silica was shown to induce red blood cells hemolysis [85] and blood monocytes injury [86]. On the other hand, surface-modified silica NPs have been successfully used for in vivo gene therapy [87] and labeling of human mesenchymal stem cells [80]. Furthermore, little is known about the possible in vivo degradation processes of these particles and the toxicity of the resulting products which limits their biomedical applications.

Although significant challenges remain in pushing this field into clinically viable therapies, there is a good reason for optimism and it is feasible that nanopharmaceuticals might become a reality in the future. By integrating nanotechnology into complex biological systems, we may achieve the detection and prevention of disease at the earliest stages of development. In addition, we can also envisage complex bio-NPs that incorporate biological functions being fully integrated into nanodevices for light/energy harvesting, biosensing or molecular electronics, opening the possibility of fabrication of truly smart bioprobes and biosensors. Overall, we have far from exhausted the biological potential of bioconjugated silica NPs. Thus, the current picture of silica NP technology is bound to evolve rapidly in medical diagnostics, targeted therapeutics, molecular biology, and cell biology, resulting in scientific and commercial opportunities that are virtually unimaginable at this time.

\section{Concluding remarks}

The demand for highly sensitive nonisotopic bioanalysis systems for biotechnology applications, such as those needed in clinical diagnostics, food quality control, and drug delivery, has driven research in the use of nanomaterials for biomedical and biotechnological applications. As one such nanomaterial, bioconjugated silica NPs have demonstrated unique advantages by virtue of their size and their novel optical, magnetic, and other properties.

As robust and bright light emitters, dye-doped silica NPs have been adopted as a new class of fluorescent labels. Six years after the first experiments on their uses in biological applications, there have been dramatic improvements in understanding surface chemistry, biocompatibility, multiplexing capability, and targeting specificity. Magnetic silica NPs have also been applied in biomolecule/ cell sorting and immunoassay in pathology laboratories. As soon as these NPs can be successfully incorporated into a complex biological system, they will have far-reaching impact in molecular biology, biotechnology, and nanomedicine.

\section{Acknowledgments}

We thank all our coworkers whose research work is reported in this paper. This work was supported by US NIH grants, NSF NIRT and State of Florida Center of Excellence for nano-biosensors.

\section{References}

[1] Niemeyer, C. M. Nanoparticles, proteins, and nucleic acids: Biotechnology meets materials science. Angew. 
Chem. Int. Ed. 2001, 40, 4128-4158.

[2] Gao, X. H; Nie, S. M. Molecular profiling of single cells and tissue specimens with quantum dots. Trends Biotechnol. 2003, 21, 371-373.

[3] Nicewarner-Pena, S. R.; Freeman, R. G.; Reiss, B. D. Submicrometer metallic barcodes. Science 2001, 294, 137-141.

[4] Rosi, N. L.; Giljohann, D. A.; Thaxton, C. S.; LyttonJean, A. K. R.; Han, M. S.; Mirkin, C. A. Oligonucleotidemodified gold nanoparticles for intracellular gene regulation. Science 2006, 312, 1027-1030.

[5] Medintz, I. L.; Uyeda, H. T.; Goldman, E. R.; Mattoussi, $\mathrm{H}$. Quantum dot bioconjugates for imaging, labeling and sensing. Nat. Mater. 2005, 4, 435-446.

[6] Smith, A. M.; Dave, S. V.; Nie, S. M.; True, L.; Gao, X. H. Multicolor quantum dots for molecular diagnostics of cancer. Expert Rev. Mol. Diagn. 2006, 6, 231-244.

[7] Atanasijevic, T.; Shusteff, M.; Fam, P.; Jasanoff, A. Calciumsensitive MRI contrast agents based on superparamagnetic iron oxide nanoparticles and calmodulin. P. Natl. Acad. Sci. USA 2006, 103, 14707-14712.

[8] Salata, O. V. Applications of nanoparticles in biology and medicine. J. Nanobiotechnol. 2004, 2, 3-8.

[9] Stroh, M.; Zimmer, J. P.; Duda, D. G.; Levchenko, T. S.; Cohen, K. S.; Brown, E. B.; Scadden, D. T.; Torchilin, V. P.; Bawendi, M. G.; Fukumura, D.; Jain, R. K. Quantum dots spectrally distinguish multiple species within the tumor milieu in vivo. Nat. Med. 2005, 11, 678-682.

[10] Michalet, X.; Pinaud, F. F.; Bentolila, L. A.; Tsay, J. M.; Doose, S.; Li, J. J.; Sundaresan, G.; Wu, A. M.; Gambhir, S. S.; Weiss, S. Quantum dots for live cells, in vivo imaging, and diagnostics. Science 2005, 307, 538-544.

[11] Mirkin, C. A.; Letsinger, R. L.; Mucic, R. C.; Storhoff, J. J. A DNA-based method for rationally assembling nanoparticles into macroscopic materials. Nature 1996, 382, 607-609

[12] Daniel, M. C.; Astruc, D. Gold nanoparticles: Assembly, supramolecular chemistry, quantum-size-related properties, and applications toward biology, catalysis, and nanotechnology. Chem. Rev. 2004, 104, 293-346.

[13] Nichkova, M.; Dosev, D.; Gee, S. J.; Hammock, B. D.; Kennedy, I. M. Microarray immunoassay for phenoxybenzoic acid using polymer encapsulated Eu: $\mathrm{Gd}_{2} \mathrm{O}_{3}$ nanoparticles as fluorescent labels. Anal. Chem. 2005, 77, 6864-6873.

[14] Chen, Y.; Chi, Y.; Wen, H.; Lu, Z. Sensitized luminescent terbium nanoparticles: preparation and time-resolved fluorescence assay for DNA. Anal. Chem. 2007, 79, 960965.

[15] Tan, W. H.; Wang, K. M.; He, X.; Zhao, X. J.; Drake, T.; Wang, L.; Bagwe, R. P. Bionanotechnology based on silica nanoparticles. Med. Res. Rev. 2004, 24, 621-638.

[16] Wang, L.; Wang, K. M.; Swadeshmukul, S.; Zhao, X. J.; Hilliard, L. R.; Smith, J.; Tan, W. H. Watching silica nanoparticles glow in the biological world. Anal. Chem. 2006, 78, 646A-654A.

[17] Yao, G.; Wang, L.; Wu, Y.; Smith, J.; Xu, J.; Zhao, W. Lee, E.; Tan, W. H. FloDots: luminescent nanoparticles. Anal. Bioanal. Chem. 2006, 385, 518-524.

[18] Trewyn, B. G.; Giri, S.; Slowing, I. I.; Lin, V. S. Mesoporous silica nanoparticle based controlled release, drug delivery, and biosensor systems. Chem. Commun. 2007, 32363245.

[19] Ow, H.; Larson, D. R.; Srivastava, M.; Baird, B. A.; Webb, W. W.; Wiesner, U. Bright and stable core-shell fluorescent silica nanoparticles. Nano Lett. 2005, 5, 113 117

[20] Ye, Z. Q.; Tan, M. Q.; Wang, G. L.; Yuan, J. L. Novel fluorescent europium chelate-doped silica nanoparticles: preparation, characterization and time-resolved fluorometric application. J. Mater. Chem. 2004, 14, 851856.

[21] Turney, K.; Drake, T. J.; Smith, J. E.; Tan, W. H.; Harrison, W. W. Functionalized nanoparticles for liquid atmospheric pressure matrix-assisted laser desorption/ionization peptide analysis. Rapid Commun. Mass Spectrom. 2004 18, 2367-2374.

[22] Hergt, R.; Dutz, S.; Müller, R.; Zeisberger, M. Magnetic particle hyperthermia: nanoparticle magnetism and materials development for cancer therapy. J. Phys.: Condens. Matter 2006, 18, S2919-S2934.

[23] Barbé, C.; Bartlett, J.; Kong, L.; Finnie, K.; Lin, H. Q.; Larkin, M.; Calleja, S.; Bush, A.; Calleja, G. Silica particles: A novel drug-delivery system. Adv. Mater. 2004, 16, 1959-1966.

[24] Wu, J.; Ye, Z. Q.; Wang, G. L.; Yuan, J. L. Multifunctional nanoparticles possessing magnetic, long-lived fluorescence and bio-affinity properties for time-resolved fluorescence cell imaging. Talanta 2007, 72, 1693-1697.

[25] Wu, C. L.; Hong, J. Q.; Guo, X. Q.; Huang, C. B.; Lai, J. P.; Zheng, J. S.; Chen, J. B.; Mu, X.; Zhao, Y. B. Fluorescent Core-shell silica nanoparticles as tunable precursors: 
Towards encoding and multifunctional nano-probes. Chem. Commun. 2008, 750-752.

[26] Yamauchi, H.; Ishikawa, T.; Kondo, S. Surface characterization of ultramicro spherical particles of silica prepared by w/o microemulsion method. Colloids Surf. 1989, 37, 71-80.

[27] Osseo-Asare, K.; Arriagada, F. J. Preparation of $\mathrm{SiO}_{2}$ nanoparticles in a non-ionic reverse micellar system. Colloids Surf. 1990, 50, 321-339.

[28] Lindberg, R.; Sjöblom, J.; Sundholm, G. Preparation of silica particles utilizing the sol-gel and emulsion-gel processes. Colloids Surf. 1995, 99, 79-88.

[29] Santra, S.; Tapec, R.; Theodoropoulou, N.; Dobson, J.; Hebard, A.; Tan, W. H. Synthesis and characterization of silica-coated iron oxide nanoparticles in microemulsion: the effect of nonionic surfactants. Langmuir 2001, 17, 2900-2906.

[30] Santra, S.; Wang, K. M.; Tapec, R.; Tan, W. H. Development of novel dye-doped silica nanoparticles for biomarker application. J. Biomed. Opt. 2001, 6, 160166.

[31] Santra, S.; Zhang, P.; Wang, K. M.; Tapec, R.; Tan, W. H. Conjugation of biomolecules with luminophore-doped silica nanoparticles for photostable biomarkers. Anal. Chem. 2001, 73, 4988-4993.

[32] Qhobosheane, M.; Santra, S.; Zhang, P.; Tan, W. H. Biochemically functionalized silica nanoparticles. Analyst 2001, 126, 1274-1278.

[33] Wang, L.; Tan, W. H. Multicolor FRET silica nanoparticles by single wavelength excitation. Nano Lett. 2006, 6, 8488.

[34] Tapec, R.; Zhao, X. J.; Tan, W. H. Development of organic dye-doped silica nanoparticles for bioanalysis and biosensors. J. Nanosci. Nanotechnol. 2002, 2, 405-409.

[35] Zhao, X. J.; Hilliard, L. R.; Mechery, S. J.; Wang, Y.; Bagwe, R. P.; Jin, S.; Tan, W. H. A rapid bioassay for single bacterial cell quantitation using bioconjugated nanoparticles. P. Natl. Acad. Sci. USA 2004, 101, 1502715032.

[36] Schmidt, J.; Guesdon, C.; Schomäcker, R. Reaction engineering aspects of the preparation of nanocrystalline particles. J. Nanopart. Res. 1999, 1, 267-276.

[37] Stöber, W.; Fink, A.; Bohn, E. Controlled growth of monodisperse silica spheres in micron size range. J. Colloid Interface Sci. 1968, 26, 62-69.

[38] van Blaaderen, A.; Imhof, A.; Hage, W.; Vrij, A. Three- dimensional imaging of submicrometer colloidal particles in concentrated suspensions using confocal scanning laser microscopy. Langmuir 1992, 8, 1514-1517.

[39] van Blaaderen, A.; Vrij, A. Synthesis and characterization of colloidal dispersions of fluorescent, monodisperse silica spheres. Langmuir 1992, 8, 2921-2931.

[40] Verhaegh, N. A. M.; van Blaaderen, A. Dispersions of rhodamine-labeled silica spheres: Synthesis, characterization, and fluorescence confocal scanning microscopy. Langmuir 1994, 10, 1427-1438.

[41] Nyffenegger, R.; Quellet, C.; Ricka, J. Synthesis of fluorescent monodisperse, colloidal silica particles. J. Colloid Interface Sci. 1993, 159, 150-157.

[42] Gerion, D.; Pinaud, F.; Williams, S. C.; Parak, W. J.; Zanchet, D.; Weiss, S.; Alivisatos, A. P. Synthesis and properties of biocompatible water-soluble silica-coated $\mathrm{CdSe/ZnS} \mathrm{semiconductor} \mathrm{quantum} \mathrm{dots.} \mathrm{J.} \mathrm{Phys.} \mathrm{Chem.}$ B 2001, 105, 8861-8871.

[43] Zhao, X. J.; Tapec-Dytioco, R.; Wang, K. M.; Tan, W. H. Collection of trace amounts of DNA/mRNA molecules using genomagnetic nanocapturers. Anal. Chem. 2003, 75, 3476-3483.

[44] Giri, S.; Trewyn, B. G.; Lin, V. S. Mesoporous silica nanomaterial-based biotechnological and biomedical. Nanomedicine 2007, 2, 99-111.

[45] Lim, M. H.; Blanford, C. F.; Stein, A. Synthesis of ordered microporous silicates with organosulfur surface groups and their applications as solid acid catalysts. Chem. Mater. 1998, 10, 467-470.

[46] Voss, R.; Brook, M. A.; Thompson, J.; Chen, Y.; Pelton, R. H.; Brennan, J. D. Non-destructive horseradish peroxidase immobilization in porous silica nanoparticles. J. Mater. Chem. 2007, 17, 4854-4863.

[47] Santra, S.; Yang, H.; Dutta, D.; Stanley, J. T.; Holloway, P. H.; Tan, W. H.; Moudgil, B. M.; Mericle, R. A. TAT conjugated FITC doped silica nanoparticles for bioimaging applications. Chem. Commun. 2004, 28102811.

[48] Wang, L.; Lofton, C.; Popp, M.; Tan, W. H. Using luminescent nanoparticles as staining probes for Affymetrix GeneChips. Bioconjugate Chem. 2007, 18, 610-613.

[49] Hermanson, G. T. Bioconjugate Techniques; Academic Press: San Diego, 1996.

[50] Roy, I.; Ohulchanskyy, T. Y.; Bharali, D. J.; Pudavar, H. E.; Mistretta, R. A.; Kaur, N.; Prasad, P. N. Optical tracking of 
organically modified silica nanoparticles as DNA carriers: A nonviral, nanomedicine approach for gene delivery. $P$. Natl. Acad. Sci. USA 2005, 102, 279-284.

[51] Zhu, S. G.; Xiang, J. J.; Li, X. L.; Shen, S. R.; Lu, H. B.; Zhou, J.; Xiong, W.; Zhang, B. C.; Nie, X. M.; Zhou, M; Tang, K; Li, G. Y. Poly(L-lysine)-modified silica nanoparticles for the delivery of antisense oligonucleotides. Biotechnol. Appl. Bioc. 2004, 39, 179-187.

[52] van Blaaderen, A.; Vrij, A. Synthesis and characterization of monodisperse colloidal organo-silica spheres. J. Colloid Interface Sci. 1993, 156, 1-18.

[53] Bagwe, R. P.; Yang, C.; Hilliard, L.; Tan, W. H. Optimization of dye-doped silica nanoparticles prepared using reverse microemulsion method. Langmuir 2004, 20, 8336-8342.

[54] Zhao, X. J.; Bagwe, R. P.; Tan, W. H. Development of organic-dye-doped silica nanoparticles in reverse microemulsion. Adv. Mater. 2004, 16, 173-176.

[55] Wang, L.; O' Donoghue, M.; Tan, W. H. Nanoparticles for multiplex diagnostics and imaging. Nanomedicine 2006, 1, 413-426.

[56] Choi, J.; Burns, A. A.; Williams, R. M.; Zhou, Z.; FleskenNikitin, A.; Zipfel, W. R.; Wiesner, U.; Nikitin, A. Y. Core-shell silica nanoparticles as fluorescent labels for nanomedicine. J. Biomed. Opt. 2007, 12, 064007.

[57] Ow, H.; Larson, D. R.; Srivastava, M.; Baird, B. A.; Webb, W. W.; Wiesner, U. Bright and stable core-shell fluorescent silica nanoparticles. Nano Lett. 2005, 5, 113117.

[58] He, X. X.; Duan, J. H.; Wang, K. M.; Tan, W. H.; Lin, X.; $\mathrm{He}, \mathrm{C}$. M. A novel fluorescent label based on organic dye-doped silica nanoparticles for HepG liver cancer cell recognition. J. Nanosci. Nanotechno. 2004, 4, 585-589.

[59] Santra, S.; Liesenfeld, B.; Dutta, D.; Chatel, D.; Batich, C. D.; Tan, W. H.; Moudgil, B. M.; Mericle, R. A. Folate conjugated fluorescent silica nanoparticles for labeling neoplastic cells. J. Nanosci. Nanotechnol. 2005, 5, 899904.

[60] Ellington, A. D.; Szostak, J. W. In vitro selection of RNA molecules that bind specific ligands. Nature 1990, 346, 818-822.

[61] Herr, J. K.; Smith, J. E.; Medley, C. D.; Shangguan, D. H.; Tan, W. H. Aptamer-conjugated nanoparticles for selective collection and detection of cancer cells. Anal. Chem. 2006, 78, 2918-2924.

[62] Smith, J. E.; Medley, C. D.; Tang, Z. W.; Shangguan, D. H.;
Lofton, C.; Tan, W. H. Aptamer-conjugated nanoparticles for the collection and detection of multiple cancer cells. Anal. Chem. 2007, 79, 3075-3082.

[63] Mechery, S. J.; Zhao, X. J.; Wang, L.; Hilliard, L. R.; Munteanu, A.; Tan, W. H. Using bioconjugated nanoparticles to monitor $E$. coli in a flow channel. Chem. Asian J. 2006, 1, 384-390.

[64] Hardiman, G. Microarray technologies 2003-An overview. Pharmacogenomics 2003, 4, 251-256.

[65] Zhao, X. J.; Tapec-Dytioco, R.; Tan, W. H. Ultrasensitive DNA detection using highly fluorescent bioconjugated nanoparticles. J. Am. Chem. Soc. 2003, 125, $11474-$ 11475.

[66] Zhou, X.; Zhou, J. Improving the signal sensitivity and photostability of DNA hybridizations on microarrays by using dye-doped core-shell silica nanoparticles. Anal. Chem. 2004, 76, 5302-5312.

[67] Wang, L.; Yang, C. Y.; Tan, W. H. Dual-luminophoredoped silica nanoparticles for multiplexed signaling. Nano Lett. 2005, 5, 37-43.

[68] Wang, L.; Zhao, W.; O' Donoghue, M.; Tan, W. H. Fluorescent nanoparticles for multiplexed bacteria monitoring. Bioconjugate Chem. 2007, 18, 297-301.

[69] Tartaj, P.; Morales, M. P.; Gonzalez-Carreno, T.; Veintemillas-Verdaguer, S.; Sernam, C. J. Advances in magnetic nanoparticles for biotechnology applications. J. Magn. Magn. Mater. 2005, 290-291, 28-34.

[70] Tyagi, S.; Kramer, F. R. Molecular beacons: Probes that fluoresce upon hybridization, Nat. Biotechnol. 1996, 14, 303-308.

[71] Tan, W. H.; Fang, X. H.; Li, J. W.; Liu, X. J. Molecular beacons: A novel DNA probe for nucleic acid and protein studies. Chem.-Eur. J. 2000, 6, 1107-1111.

[72] Ravi Kumar, M. N. V.; Sameti, M.; Mohapatra, S. S.; Kong, X.; Lockey, R. F.; Bakowsky, U.; Lindenblatt, G.; Schmidt, H.; Lehr, C. M. Cationic silica nanoparticles as gene carriers: Synthesis, characterization and transfection efficiency in vitro \& in vivo. J. Nanosci. Nanotechnol. 2004, 4, 876-881.

[73] Roy, I.; Ohulchanskyy, T. Y.; Pudavar, H. E.; Bergey, E. J.; Oseroff, A. R.; Morgan, J.; Dougherty, T. J.; Prasad, P. N. Ceramic-based nanoparticles entrapping water-insoluble photosensitizing anticancer drugs: A novel drug-carrier system for photodynamic therapy. J. Am. Chem. Soc. 2003, 125, 7860-7865.

[74] Mary Ann Liebert, Inc. Assessment of adenoviral vector 
safety and toxicity: report of the National Institutes of Health Recombinant DNA Advisory Committee. Hum. Gene Ther. 2002, 13, 3-13.

[75] Muruve, D. A. The innate immune response to adenovirus vectors. Hum. Gene Ther. 2004, 15, 11571166.

[76] Lai, C. Y.; Trewyn, B. G.; Jeftinija, D. M.; Jeftinija, K.; Xu, S.; Jeftinija, S.; Lin, V. S. A mesoporous silica nanospherebased carrier system with chemically removable CdS nanoparticle caps for stimuli-responsive controlled release of neurotransmitters and drug molecules. J. Am. Chem. Soc. 2003, 125, 4451-4459.

[77] Jin, S.; Ye, K. M. Nanoparticle-mediated drug delivery and gene therapy. Biotechnol. Prog. 2007, 23, 32-41.

[78] Mohr, R.; Kratz, K.; Weigel, T.; Lucka-Gabor, M.; Moneke, M.; Lendlein, A. Initiation of shape-memory effect by inductive heating of magnetic nanoparticles in thermoplastic polymers. P. Natl. Acad. Sci. USA 2006, 103, 3540-3545.

[79] Levy, L.; Sahoo, Y.; Kim, K. S.; Bergey, E. J.; Prasad, P. N. Nanochemistry: Synthesis and characterization of multifunctional nanoclinics for biological applications. Chem. Mater. 2002, 14, 3715-3721.

[80] Lu, C.; Hung, Y.; Hsiao, J. K.; Yao, M.; Chung, T. H.; Lin, Y. S.; Wu, S. H.; Hsu, S. C.; Liu, H. M.; Mou, C. Y; Yang, C. S.; Huang, D. M.; Chen, Y. C. Bifunctional magnetic silica nanoparticles for highly efficient human stem cell labeling. Nano Lett. 2007, 7, 149-154.
[81] Santra, S.; Bagwe, R. P.; Dutta, D.; Stanley, J. T.; Walter, G. A.; Tan, W. H.; Moudgil, B. M.; Mericle, R. A. Synthesis and characterization of fluorescent, radio-opaque and paramagnetic silica nanoparticles for multimodal bioimaging applications. Adv. Mater. 2005, 7, 21652169.

[82] Kircher, M. F.; Mahmood, U.; King, R. S.; Weissleder, R.; Josephson, L. A multimodal nanoparticle for preoperative magnetic resonance imaging and intraoperative optical brain tumor delineation. Cancer Res. 2003, 63, 81228125.

[83] Lu, Y.; Yin, Y.; Mayers, B. T.; Xia, Y. Modifying the surface properties of superparamagnetic iron oxide nanoparticles through a Sol-Gel approach. Nano Lett. 2002, 2, 183186.

[84] Jiang, W.; Kim, B. Y. S.; Rutka, J. T.; Chan, W. C. W. Nanoparticle-mediated cellular response is sizedependent. Nat. Nanotechnol. 2008, 3, 145-150.

[85] Gerashchenko, B. I.; Gun'ko, V. M.; Gerashchenko, I. I.; Mironyuk, I. F.; Leboda, R.; Hosoya, H. Probing the silica surfaces by red blood cells. Cytom. Part A 2002, 49, 5661.

[86] Pomeroy, C.; Filice, G. A. Effect of intravenous silica on the course of Nocardia asteroids pneumonia. Infect. Immun. 1988, 56, 2507-2511.

[87] Luo, D.; Saltzman, W. M. Nonviral gene delivery: Thinking of silica. Gene Ther. 2006, 13, 585-586. 